\title{
Creencias, práctica docente y propuestas para la atención de la diversidad cultural: el caso de una docente costarricense y tres infantes nicaragüenses
}

\author{
Beliefs, Teaching Practice and Proposals for the Attention of Cultural \\ Diversity: the Case of a Costa Rican Teaching and Three Nicaraguans Infants
}

\author{
Lillian Susana Ruiz Guevaral \\ División de Educación Básica \\ Centro de Investigación y Docencia en Educación \\ Universidad Nacional \\ Heredia, Costa Rica \\ susanaruiz@racsa.co.cr
}

Recibido 19 de abril de 2010 • Aceptado 24 de junio de 2010

\begin{abstract}
Resumen. Este artículo presenta los datos obtenidos en un proceso de investigación acerca de las creencias de una docente en relación con tres estudiantes de origen nicaragüense. Específicamente se presentan los datos de la categoría de análisis sobre el manejo de la práctica docente y su relación con las propuestas de educación en diversidad. Para el desarrollo de esta categoría se incluye un análisis de las subcategorías que se indican a continuación: Dinámica del trabajo diario de una maestra que trabaja con infantes de otra cultura, evaluación de los estudiantes en el salón de clases, estrategias para el desarrollo de la disciplina con niños, y ereencias y propuestas en diversidad.

Los datos evidencian que, en la práctica de aula, la maestra no demuestra un trato diferenciado entre niños nicaragüenses y costarricenses, lo que hace pensar que el trato igualitario es la estrategia "per se utilizada por la docente para resolver el tema de la diversidad cultural en el aula. Por otra parte, la disciplina es asumida por la docente, en su práctica de trabajo diario, como un asunto que se aborda con criterios de regaño, utilizando tonos de voz fuerte y vocabulario agresivo.
\end{abstract}

Palabras clave. Creencias, práctica docente, atención a la diversidad, interculturalidad, metodología, disciplina, planificación.

Abstract. This article presents data from a research that studied a teacher belief regarding three Nicaraguan students. Specifically, presents findings related to teaching practice and its relation to diverse education proposals. It includes an analysis of four subcategories: daily dynamic of a teacher who works with infants of another culture, students' assessment in the classroom, discipline strategies to children, and diverse beliefs and proposals.

The data shows that, in the classroom, the teacher does not demonstrate different treatment to Nicaraguan and Costa Rican children, suggesting that equal treatment is the strategy per se used by the teacher to solve the issue of cultural diversity in the classroom. On the other hand, discipline is assumed by the teacher with scolding criteria, using a loud tone of voice and aggressive vocabulary.

Key words. Beliefs, teaching practice, attention to diversity, interculturalism, methodology, discipline, planning.

Licenciada en Educación Preescolar de la Universidad Nacional de Costa Rica, Máster en Administración Educativa de la Universidad de La Salle y Doctora en Educación de la Universidad Estatal a Distancia, Costa Rica. Académica de la División de Educación Básica de la Universidad Nacional de Costa Rica. Investigadora de temáticas relacionadas con la transición de la primaria a la secundaria, acreditación de carreras de Educación, creencias en la práctica docente. Dirección postal: Heredia, Costa Rica, Los Ángeles de San Rafael, 400 norte Super Los Ángeles, casa mano izquierda. Número de teléfono o celular: (506) 22676495, (506) 88447841. 


\section{Introducción}

Desde la perspectiva de Holingworth (citado por, Luft, 1999), desconocer las creencias de los docentes puede ser uno de los principales factores que permiten perpetuar las prácticas educativas, especialmente, aquellas consideradas como poco efectivas.

Una práctica educativa poco efectiva en el ámbito de la diversidad cultural puede definirse como aquella que dista de las nuevas propuestas relacionadas con principios de un enfoque intercultural.

El enfoque intercultural va más allá de la coexistencia de culturas distintas y se centra en el diálogo y la interacción cultural en un plano de igualdad real, lo que implica una reflexión y acción, también relación, sobre los factores ideológicos y estructurales que conllevan desigualdad y racismo. (Besalú, 2002, p. 66)

Por otra parte, Bueno (1997) sugiere que la educación intercultural se presenta como un enfoque en busca del diálogo entre las personas de diferentes culturas para una convivencia adecuada. Este planteamiento se aprecia en el siguiente párrafo:

La educación intercultural apunta a una solución al problema que se plantea en las sociedades multiculturales, ya que se refiere a una relación activa entre las culturas para llegar a desarrollar una sociedad intercultural. Por tanto, este tipo de educación pone el énfasis en los puntos de contacto y en el diálogo que se debe producir entre las culturas, siempre en un clima de igualdad y respeto. (p. 7)

\section{Referencia teórica}

\subsection{Concepto y características de la multiculturalidad e interculturalidad}

El fenómeno de la educación multicultural e intercultural es, para muchos autores, un reto que la sociedad y, por supuesto, la educación del siglo XXI no puede obviar, especialmente en el proceso de formación docente. En este sentido, Ander-Egg (2001) indica que es necesario revisar el currículo oculto que subyace en las prácticas de aula, debido a que los lineamientos teóricos curriculares vigentes están cargados de las ideologías y creencias que los docentes construyen.

Precisamente el bagaje ideológico es el que causa, en la actualidad -tal y como lo afirma Jordán (1994)-, que el concepto de educación multicultural sea asumido de manera simple, es decir, muchos docentes no han profundizado en la riqueza y trascendencia que encierra esta propuesta educativa. Además, sugiere que la concepción del maestro en muchas ocasiones está cargada de matices ideológicos humanistas, especialmente en relación con valores como la tolerancia y el respecto; sin embargo, afirma este mismo autor que los docentes no avanzan hacia un entendimiento y abordaje pedagógico favorecedor de su adaptación a las diferencias culturales de los niños provenientes de grupos étnicos variados.

En este mismo sentido, Sachs (citado por Jordán, 1994) afirma que los maestros, en su mayoría, asumen conceptos de cultura y de educación multicultural, asociados a visiones humanistas “(...) sin preguntarse apenas acerca de la cultura y de la educación multicultural en su perspectiva más pragmática-social" (p. 18). A este respecto, Jordán (1994) indica que el pensamiento de los docentes 
frente a la educación multicultural se caracteriza por matices románticos y poco prácticos, lo cual puede asociarse a la casi nula reflexión que sobre el pensamiento pedagógico ellos asumen.

En un estudio realizado por Agudo, Jaurena y Mata (2008), sobre las necesidades de formación intercultural del profesorado en España, las autoras se plantearon como uno de los dilemas a considerar "(...) la revisión de creencias y prácticas vs entrenamiento en técnicas y metodologías". Entre sus conclusiones, se indica que las instituciones encargadas de formar docentes en la actualidad han demostrado ser altamente conservadoras y hasta lentas, por cuanto "Las creencias y modelos de prácticas en las que los profesores son formados son homogéneas como lo eran hace treinta años" (p. 284). Esta preocupación de las autoras por el tema de las creencias y su repercusión en la práctica docente fue también tema de discusión para muchos investigadores quienes, en la década de los años noventa, se dedicaron a trabajar el tema y coincidieron en que... "Muchos intentos de reforma en el pasado han ignorado el rol de las creencias de los maestros en el status quo, asimismo, las creencias de los maestros y las maestras son un ingrediente crítico en los factores, que determinan lo que pasa en las aulas" (Tippins y Gallard, citados por Lumpe, Haney y Czerniak 2000, p. 276).

Por otra parte, Nespor, Pajares y Richardson (citados por Spanneberg, 2001) indican que los informes acerca de investigaciones relacionadas con las creencias de los maestros aseguran que estas influyen en la práctica de aula, como una acción consecuente de la intervención de las creencias en los juicios y percepciones que tienen los docentes. En esta misma dirección, Pajares (1992) reitera que las creencias en relación con la escuela y la enseñanza se establecen muy temprano en la vida de las personas y esto se logra por medio de las experiencias de la escolarización.

Desde otra perspectiva pero en relación con la influencia de las creencias en la práctica docente, Suck (1997) advierte que los educadores no se dan cuenta del poder y de la tenacidad de sus creencias y actitudes, razón por la cual, identificar de qué manera afectan o intervienen en sus labores educativas diarias no ha sido reconocido con claridad.

Considerar el poder de las creencias en el trabajo docente, especialmente porque estas surgen en el plano individual y se perpetúan en el plano social, es un requerimiento ineludible para el trabajo de la diversidad cultural y el cambio que se espera en el siglo XXI. En este sentido, Jordán (citado por Agudo, Jaurena y Mata, 2008) señala que se requieren cambios en la personalidad del docente para enfrentar la diversidad cultural. Estos cambios se refieren a "(..) sustituir una visión problematizadora por otra que reconoce la diversidad como normalidad, adoptar un talante reflexivo y crítico-constructivo respecto a inercias y rutinas, replantearse la tarea docente como algo más educativo que instructivo, asumir una responsabilidad ético-profesional, un compromiso, disponerse al trabajo conjunto y cooperativo con otros profesionales.” (p. 284).

Tal y como lo plantea Viñas (citado por Essomba et al., 1999), la educación intercultural es una realidad que definitivamente exige ciertos cambios en la vida y en la rutina de las aulas. Además, afirma:

Es cierto, que la globalización cultural es un proceso que a pesar de las tensiones que produce, aparece como imparable, y al cual el sistema educativo debe responder con instrumentos y una mentalidad nueva. Por tanto la educación intercultural no sólo debe percibirse en aquellos centros que tengan alumnos con diversidad de origen muy heterogéneo, sino que es una necesidad general del sistema educativo. (p. 57)

Por lo tanto, las prácticas docentes deben abordar el tema de la diversidad cultural como un asunto de urgencia y no como una posibilidad que se les presenta. Sin embargo, la construcción 
de propuestas coherentes y participativas es el elemento básico requerido en el camino hacia la integración verdadera de los niños provenientes de otras culturas.

Relacionado con los planteamientos del párrafo anterior, Viñas (citado por Essomba et al. 1999) sugiere que, en el marco general para la organización de la educación intercultural del centro, se requiere trabajar sobre una serie de ámbitos indicados en el cuadro siguiente:

Cuadro 1

Ámbitos y acciones que se requieren para organizar la educación intercultural en la institución educativa

\section{Ámbitos}

\section{Institucional}

- Definir en el proyecto educativo de centro (PEC) principios y valores en relación con la educación intercultural.

- Criterios aprobados sobre los procedimientos de adaptación de los alumnos. El plan de acogida.

- Determinar los órganos para velar por el tratamiento del alumnado en sus procesos de adaptación y seguimiento.

- Determinar específicamente las responsabilidades tutoriales.

\section{Administrativos}

- Facilitar y simplificar los procesos administrativos para la escolarización de alumnos inmigrantes o de culturas no mayoritarias.

- Facilitar, en la medida de las posibilidades del centro, el acceso de los alumnos a ayudas para la obtención de materiales académicos.

\section{Curricular}

- Criterios de coordinación del profesorado con respecto al tratamiento de los temas relacionados con la educación intercultural.

- Determinación y valoración de las necesidades curriculares de los alumnos.

- Dotar de instrumentos para ejercer la tutoría individual y en grupo.

- Determinación de criterios pedagógicos para el tratamiento de la diversidad.

- Criterios acerca de material y recursos adecuados.

- Criterios respecto a itinerarios curriculares.

- Criterios respecto a la evaluación.

- Potenciación del uso instrumental básico de las habilidades lingüísticas en la comunicación en los alumnos con dificultades.

\section{Servicios}

- Coordinación de actuaciones con profesionales externos al centro.

- Facilitar contactos con personas que puedan ejercer de mediadoras entre las familias y los centros educativos.

- Facilitar la participación de todos los alumnos en actividades complementarias y extraescolares.

\section{Relacional}

- Revisión de actitudes y pensamiento del profesorado respecto de la educación intercultural.

- Revisión de actitudes y pensamiento del alumnado con respecto a la educación intercultural

- Potenciación de la formación e información del profesorado en relación con la educación intercultural.

Nota: Viñas, J. (citado por Essomba, et al., 1999). 
Del cuadro anterior, se concluye que la educación intercultural requiere de cambios en el ámbito institucional, que abarcan desde aspectos administrativos hasta, por supuesto, los curriculares; es decir, que la integración real de estudiantes de diferentes culturas es un asunto que amerita procesos importantes de reflexión sobre la práctica docente.

En relación con este proceso de integración de un proyecto intercultural para la atención de la diversidad, es importante evaluar el tratamiento de la diversidad cultural, para lo cual Sabariego (citada por Essomba et al. 1999) indica los siguientes criterios, presentados a manera de preguntas:

a. ¿Se concede atención a la expresión de la multiculturalidad en el ambiente material del aula?

b. ¿El clima de aprendizaje es respetuoso, reconoce y acepta las diversas culturas presentes en el aula (fomentando la valoración positiva de la diversidad, así como las actitudes de respeto, tolerancia y solidaridad entre el alumnado)?

c. ¿Es posible la expresión espontánea de la propia identidad cultural? ¿Se permite que esta expresión se legitime, se valore y se analice críticamente? ¿Se permite construir la propia identidad cultural de un modo enriquecedor adquiriendo, al mismo tiempo, competencia para tener conciencia de los esquemas culturales que hay alrededor?

d. ¿Las programaciones y la selección de contenidos y materiales se efectúan con criterios de diversidad cultural?

e. ¿Se facilita la expresión oral de las vivencias culturales y de los saberes prácticos del alumnado?

f. ¿El modelo educativo que existe en el aula estimula aquellas metodologías que posibilitan el marco de interrelaciones (es decir, propuestas que favorezcan la interacción entre iguales, el trabajo cooperativo, las dinámicas comunicativas, el intercambio de roles o las tareas colectivas, de cara a facilitar el descubrimiento de la dimensión positiva del otro y fomentar relaciones interculturales constructivas)?

g. ¿Los recursos metodológicos que se utilizan permiten el tratamiento adecuado de la diversidad (la adaptación al ritmo de aprendizaje y el seguimiento personalizado del alumnado mediante una organización flexible del tiempo y la diversificación de técnicas, modalidades de trabajo, materiales, así como de actividades de aprendizaje para la adquisición de un mismo contenido y de contenidos para un mismo objetivo)?

h. ¿Se favorece un proceso de enseñanza-aprendizaje dialógico, interactivo y con más refuerzos positivos que negativos?

i. ¿En la resolución de conflictos se utilizan el diálogo crítico y el debate como medidas preferentes? (p. 30-31)

Estas interrogantes que plantea Sabariego (citada por Essomba et al., 1999) pueden utilizarse, también, para la evaluación de la práctica docente de los maestros y las maestras, quienes, en un esfuerzo por tener parámetros que les permitan mayor certeza de su trabajo, en múltiples ocasiones -indica la bibliografía-, asumen conceptos "light" o de trato igualitario, para la atención de la diversidad cultural. 


\section{Metodología}

\subsection{Tipo de investigación}

Debido a que uno de los objetivos generales de la investigación que respalda el presente artículo se refiere a analizar la relación entre la práctica docente de una maestra costarricense que atiende el ciclo de transición del jardín público Luces y las propuestas educativas para la atención de la diversidad cultural, la investigadora decide que este estudio se realice desde el enfoque de investigación cualitativa. Esta decisión se justifica en la necesidad de abordar las vivencias y creencias desde la perspectiva de los actores mismos (docentes y estudiantes). Con base en ello, se determina utilizar el método etnográfico, para así incursionar en el campo de trabajo e interactuar con la docente y los estudiantes, especialmente con los de origen nicaragüense. Es oportuno aclarar que este método de trabajo exigió la recolección de evidencias tales como fotografías, dibujos de los estudiantes, información básica de los estudiantes registrada en sus expedientes, los planes de clase de la docente, entre otra información. Con el objetivo de conocer la práctica de trabajo diario y el papel que desempeñaron sus actores, se utiliza la observación participante como técnica primordial de recolección de información.

El diseño metodológico de la presente investigación se caracterizó, también, por la utilización del método de estudio de casos, especialmente porque la temática requirió explorar la particularidad de las creencias de una maestra, en relación con el desarrollo de tres infantes nicaragüenses, como principal eje de la investigación. Lo anterior no omite que los infantes costarricenses actuaron dentro del contexto de aula y, por tanto, sus opiniones también fueron tenidas en cuenta.

\subsection{Participantes del proceso de investigación}

En la presente investigación, el estudio de casos se refiere a la situación específica de una docente de preescolar, una niña y dos niños nicaragüenses, quienes asisten al ciclo de transición. Es oportuno especificar que, para la selección de los estudiantes, se consideró como criterio que fueran nacidos en Nicaragua y que sus padres mantuvieran vínculos con este país de origen (por medio de visitas realizadas dos años previos, como máximo, al desarrollo de la investigación).

En términos específicos, la docente del kínder 2 (nombre del grupo de transición) es una profesional con un bachillerato universitario y una experiencia laboral de 15 años.

En relación con la niña y los dos niños, para guardar su identidad, se les asignó el código de participante K, R y S, respectivamente. Como ya se anotó, los tres infantes son de nacionalidad nicaragüense.

Por otra parte, es oportuno agregar que el grupo K2 del ciclo de transición estuvo compuesto también por 23 niños de origen costarricense, a quienes se les asignó un rol menos activo dentro del proceso de investigación, por el interés particular de la investigación de enfocarse en los infantes nicaragüenses y la docente costarricense.

Las categorías de análisis que permitieron el desarrollo del estudio surgen, en un primer momento, de la búsqueda teórica relacionada con la temática investigada; sin embargo, el ingreso al campo y el trabajo en él orientan a la investigadora para reelaborar estos planteamientos iniciales. Para este artículo se presentan los datos relacionados con dos de las categorías abarcadas en la investigación. 


\section{Las creencias del manejo de la práctica docente y su relación con las propuestas de educación en diversidad}

Incluye un análisis de las siguientes subcategorías:

a. Dinámica del trabajo diario de una maestra que trabaja con infantes de otra cultura

b. La evaluación de los estudiantes en el salón de clases

c. Estrategias para el desarrollo de la disciplina con niños

d. Creencias y propuestas en diversidad

Las técnicas e instrumentos utilizados por la investigadora, para el desarrollo de la investigación que antecede este artículo se incluyen en la siguiente tabla. Estas técnicas e instrumentos buscan la triangulación de los datos por fuente de información.

Tabla 1

Técnicas e instrumentos utilizados para el desarrollo de la investigación

\begin{tabular}{|c|c|c|}
\hline Técnica & Instrumento & Participante a quien se aplica \\
\hline - Observación participante. & $\begin{array}{l}\text { Matriz de observaciones: nota } \\
\text { cruda, cocida y teórica }\end{array}$ & $\begin{array}{l}\text { Docente, niños nicaragüienses y } \\
\text { costarricenses }\end{array}$ \\
\hline \multirow[t]{2}{*}{ - Entrevista } & $\begin{array}{l}\text { a. Conversaciones informales } \\
\text { b. Entrevista semiestructurada } \\
\text { c. Frases abiertas }\end{array}$ & $\begin{array}{l}\text { a. Docente y niños } \\
\text { b y c. Total de niños del } \\
\text { aula (costarricenses y } \\
\text { nicaragüenses) }\end{array}$ \\
\hline & d. Entrevistas en profundidad & $\begin{array}{l}\text { d. Docente y niños } \\
\text { nicaragüenses y } \\
\text { costarricenses }\end{array}$ \\
\hline - Fotografías & $\begin{array}{l}\text { Matriz: Fotografía, descripción, } \\
\text { evento, categoría y análisis }\end{array}$ & $\begin{array}{l}\text { Docente y total de niños que } \\
\text { asisten al salón (nicaragüenses y } \\
\text { costarricenses }\end{array}$ \\
\hline $\begin{array}{l}\text { - } \quad \text { Análisis de documentos: } \\
\text { - Expediente de niños nicaragüenses } \\
\text { - Cuaderno de apresto de niños } \\
\text { nicaragüenses: }\end{array}$ & $\begin{array}{l}\text { Matriz: Área de desarrollo, } \\
\text { Descripción de actividad, valoración } \\
\text { de logro y categoría de análisis }\end{array}$ & No aplica \\
\hline
\end{tabular}

Nota. Ruiz, 2005 Tesis para optar por el grado de doctorado en educación

La decisión de utilizar varias técnicas e instrumentos para la recolección de la información se relacionó directamente con la necesidad de triangular los datos como estrategia de validación de la información. Lo anterior, ya que según Maxwell (1996), en los estudios cualitativos es útil recurrir a la triangulación, la cual implica recopilar información desde diferentes individuos y contextos, utilizando una variedad de instrumentos, como por ejemplo: diarios, entrevistas formales e informales, entre otros. 


\subsection{Triangulando información: La alternativa para el análisis del dato}

En términos de la estrategia para el análisis de los datos, esta implicó un proceso de análisis y de síntesis constante; es decir, un ir y venir, producto de la contrastación y comparación de los datos. Por otra parte, es importante indicar que el análisis de datos se inició desde que la investigadora ingresó al campo, para así ser coherente con la metodología etnográfica.

Una vez realizadas las observaciones y aplicados los instrumentos, la información se despliega en matrices para iniciar el análisis de las categorías establecidas y las emergentes. Concluido este proceso, se triangulan los datos, para lo cual se utiliza la siguiente matriz.

Tabla 2

Triangular el dato por fuente de información

\begin{tabular}{ll}
\hline Fuente o instrumento de observación & Datos \\
\hline Información de observaciones & \\
Información de entrevistas & \\
Información de conversaciones & \\
Información de expedientes \\
Dato vivo (fotografías y dibujos del apresto) \\
Cuadros referentes a instrumento de frases abiertas
\end{tabular}

\subsection{Fases del diseño de la presente investigación}

La investigación incluyó 4 etapas. Una de preparación, para fortalecer las preguntas de investigación e iniciar el vagabundeo teórico. En la segunda etapa se ingresó al campo para iniciar un proceso de negociación para la elección de los casos a seguir, además, se inició un proceso de observación constante. En la etapa analítica e interpretativa, los datos se reducen por medio de la codificación y el levantado de matrices que se nutren de los protocolos de observación (26 en total). En la última etapa se construye el informe final.

\section{Resultados y discusión}

Los datos que se incluyen a continuación permiten valoran la práctica docente de una maestra que trabaja con tres niños nicaragüenses y 23 costarricenses. Para esta valoración se consideran elementos tales como la metodología de trabajo, disciplina y evaluación en la dinámica de trabajo diario.

Es importante indicar que la existencia de niños y niñas de otras culturas y nacionalidades en un centro educativo y en una aula exige, necesariamente, por parte de la dirección y la docente, aplicaciones prácticas acordes con las demandas de dicha población, sin que ello implique, un proyecto multicultural y/ o intercultural en la institución, aunque esto sería muy importante. Sin embargo, por conversaciones sostenidas con la docente $\mathrm{N}$, es evidente que la institución donde 
se realiza la investigación no cuenta, de forma explícita, con un proyecto de este tipo; es decir, que se realizan acciones aisladas para facilitar a los niños y las niñas nicaragüienses su ingreso y permanencia al sistema escolar. Por ejemplo, se les admite, aunque no presenten sus partidas de nacimiento a tiempo y la comunicación con los padres y madres de familia sea escasa y cortante, especialmente porque, en general, no tienen sus papeles en regla y, por tanto, se encuentran en calidad de ilegales.

Lo cierto es que la docente $\mathrm{N}$ conoció de la existencia de una niña y dos niños nicaragüenses y supo que debía trabajar con ella y ellos al iniciar el curso lectivo; no obstante, su práctica docente -tal y como ella misma lo expresó- no pudo ubicarla o relacionarla necesariamente con alguna propuesta para la atención de la diversidad (multicultural o intercultural) o perspectiva teóricopráctica. Aún así, es necesario proceder a la caracterización de la práctica educativa de la docente $\mathrm{N}$ y su relación con dichas perspectivas, para conocer la realidad de este salón en términos de la educación intercultural.

\subsection{La dinámica de trabajo diario como espejo de la improvisación}

En términos de planificación, existen una serie de ámbitos que deben considerarse para la organización de la educación intercultural en el centro educativo y que se refieren a:

Criterios de coordinación de la profesora con respecto al tratamiento de los temas relacionados con la educación intercultural.

Determinación y valoración de las necesidades curriculares de los alumnos.

Dotación de instrumentos para ejercer la tutoría individual y en grupo.

Determinación de criterios pedagógicos para el tratamiento de la diversidad.

Criterios sobre material y recursos adecuados.

Criterios respecto a itinerarios curriculares.

Criterios respecto de la evaluación. (Jordán, citado por Essomba et.al., 1999, p. 62)

En cuanto a la práctica educativa de la docente $\mathrm{N}$, se establece que ella fue quien seleccionó los temas de trabajo (los cuales fueron pocos y su planificación improvisada en la mayoría de los casos). Es decir, se obvió la participación de la niña y los niños nicaragüienses, así como sus necesidades e intereses. Por otra parte, del total de 26 observaciones realizadas, no se logró identificar temáticas o actividades que procuraran el desarrollo de temas relacionados con la realidad intercultural que se vivenció en el salón de clases.

Por otra parte, en términos de coordinación de la docente $\mathrm{N}$ con el grupo de docentes de la institución y del nivel de preescolar propiamente, ella indicó que ... "La forma de planear que nosotras tenemos es con las compañeras en grupo, es decir, una vez cada quince días nos reunimos las que queremos y lo hacemos juntas, los temas los elegimos según el programa para que nada se nos quede sin cubrir y luego trabajamos con los niños, pero ellos no participan directamente". (Registro de Conversación informal 7, 2005)

Según lo anterior, existió coordinación de la docente $\mathrm{N}$ con las compañeras de nivel de preescolar de la institución. Por otra parte, de acuerdo con consulta realizada directamente a ella, indica que... "La institución solamente realiza actividades especiales como actos cívicos para fechas especiales que se encuentran planificadas en el calendario escolar, pero temas relacionados con temas interculturales no, la verdad no" (Registro de observación 9, 2005). 
Por lo tanto, se logra establecer que la coordinación que realizó la docente $\mathrm{N}$ se refirió a la actividad específica de planeamiento; pero en el aula y en la institución no se identificaron temas relacionados con la interculturalidad, por tanto, el 100\% de la población infantil nicaragüense que asistió a este centro educativo, el cual representó alrededor del $40 \%$ del total de la población estudiantil de la institución, no fue atendido en términos reales de sus necesidades sociales y culturales, así como de integración real; situación que también afectó, también, a la población costarricense, la cual perdió su posibilidad de enriquecerse culturalmente.

Otro de los criterios teóricos mencionados en párrafos anteriores sugiere que se deben considerar las necesidades curriculares de la alumna y los dos alumnos nicaragüenses, para la construcción de un aula tendiente a la promoción de la interculturalidad. Pese a ello, tal y como se constató en los protocolos de observación, la práctica docente de la maestra participante se caracterizó por una ausencia de planificación clara en torno a temas específicos a desarrollar. Solamente se observaron evidencias de cuatro temas, a saber: los números, las festividades patrias (15 de setiembre), el adulto mayor y el sistema solar.

Frente a la situación anterior, en la entrevista aplicada a los niños nicaragüenses y costarricenses del K2, se les consultó acerca de su opinión en relación con los temas que se habían trabajado hasta el momento. Del total de infantes consultados, un $45 \%$ insistió en que eran aburridos y cansados; el 25\% indicó que su disgusto con los temas, porque no jugaron o cantaron; un 15\% sugirió que le gustó el tema del sistema solar, porque ellos expusieron; el otro 15\% afirmó no acordarse de los temas trabajados, y un 5\% dijo que fueron temas muy bonitos; sin embargo, al tratar de profundizar en esta afirmación, los niños y las niñas no logran justificar qué fue lo que les agradó.

En relación con los niños nicaragüenses, en la entrevista realizada se les consultó acerca de la posibilidad de trabajar temas de Nicaragua. Algunas de las sugerencias que ella y ellos ofrecen evidencian su necesidad de que los compañeros costarricenses conozcan las riquezas de su país de origen. En las siguientes frases de la niña y los niños nicaragüenses, puede apreciarse lo anteriormente planteado:

$\mathrm{K}$ : "Yo quiero que mis compañeros conozcan de lo que comemos, porque mi mamá dice que nosotros cocinamos muy rico".

R: "Yo quiero que mis compañeritos conozcan dónde vivo en Nicaragua y dónde viven mis amigos, además allá nosotros tenemos playas muy bonitas y ellos (compañeros) pueden verlas en fotos y se animen a ir para allá".

S: "Yo quiero que hablemos del béisbol, porque en Nicaragua dice mi papa, que jugamos muy bien y aquí casi no se oye solo de fútbol".

(Entrevista aplicada a los niños nicaragüenses)

Frente a estas sugerencias que plantean la niña y los dos niños nicaragüenses, la docente $\mathrm{N}$ expresó que eran muy interesantes y que quizás para otras fechas se podrían rescatar:

Docente N: "Que vacilón lo que dicen, yo creo que ellos son muy inteligentes y tal vez para el 12 de octubre se pueda retomar lo que ellos piden, tengo que apuntarlo y tenerlo presente" (Registro Devolución de información 6, 2005).

En términos generales, los intereses y necesidades de la población nicaragüense, e inclusive la costarricense, no se consideran al momento de realizar las planificaciones de los temas. Planificaciones que, a su vez, tienen rasgos de improvisación constante, lo cual hace suponer una 
ausencia de sistematización de la práctica docente, confirmada en la variedad de lugares que utiliza la docente $\mathrm{N}$ para apuntar las áreas a las cuales asisten los infantes, negando la importancia de un proceso de desarrollo integral. De ahí que la creencia implícita "el juego es para los niños" se comienza a explicitar con fuerza en este trabajo.

En lo relativo a criterios de materiales, horario y evaluación, la práctica docente sugiere que estos se rigen por las disposiciones del Ministerio de Educación (MEP); es decir, que el horario, por ejemplo, se organizó tomando en consideración uno de los módulos del MEP y este, a su vez, fue diseñado por la dirección administrativa de la institución. Los materiales, por otra parte, no se variaron durante el año, tal y como se evidencia en las descripciones del salón de clases para el I y II semestres del curso lectivo.

En el caso de la información anterior, se evidencia la no realización de modificaciones o bien la introducción de espacios para que la niña y los dos niños nicaragüenses pudieran dar a conocer sus vivencias culturales. Por el contrario, en este caso parece que el mismo Ministerio de Educación Pública asumió una labor controladora que no facilitó la promoción de políticas y cambios en las rutinas que pudieron ser aprovechadas por la docente $\mathrm{N}$ y la institución en general.

\subsection{Evaluación: un elemento para la homogeneización de potencialidades}

En lo relativo a instrumentos de evaluación, la docente $\mathrm{N}$ realizó una observación por trimestre para cada uno de los tres infantes nicaragüenses. Además, a principio de año les aplicó a todos sus estudiantes (costarricenses y nicaragüenses) el diagnóstico inicial elaborado en la institución en conjunto con las compañeras de nivel y que, en términos de estructura, se orientó a la identificación de contenidos generales y previos en la niña y el niño; contenidos que se refirieron a números, colores, figuras geométricas, esquema de figura humana, habilidad para el recorte, relaciones espaciales, entre otros.

Posteriormente, al finalizar el curso lectivo, la docente $\mathrm{N}$ aplicó la evaluación utilizando un instrumento elaborado por el MEP y la Asesoría de Preescolar, titulado Control de Logros, en el que se incluyó un listado tipo escala Likert de habilidades y destrezas, que se evalúa siguiendo comandos tales como Lo logra (LL), En proceso (EP) y Necesita Ayuda (NA). Este instrumento, al igual que el de diagnóstico, evalúa el logro de contenidos básicos como números, colores, relaciones temporo-espaciales, figuras geométricas, entre otros; sin embargo, no se identificaron elementos que sugieran procesos o bien el reconocimiento de potencialidades en los infantes nicaragüenses.

Por otra parte, en ninguno de los instrumentos utilizados por la docente $\mathrm{N}$ se incluyeron elementos que permitieran dar a conocer la riqueza cultural en su salón de clases, ni tampoco las potencialidades de sus estudiantes. Este hecho manifestó una escasa preocupación por la diversidad como un asunto propio del ser humano y de la interculturalidad como propuesta educativa.

Tal y como lo indica Jordán (citado por Essomba et.al, 1999), uno de los ámbitos que debe considerarse para la organización de la educación intercultural en el centro educativo se refiere a la determinación de "(...) criterios pedagógicos para el tratamiento de la diversidad" (p. 62). Sin embargo, la construcción de los diagnósticos y la elección del instrumento de evaluación evidencian su ausencia en el tratamiento de la diversidad. Esto debido a que se homogeniza a la niña y los dos niños nicaragüenses por igual, sin que se intente considerar sus conocimientos previos.

Por otra parte, los criterios pedagógicos de los cuales habla Jordán (citado por Essomba et al., 1999) se evidenciaron en la práctica propiamente dicha, por cuanto las actividades de aula que se identificaron, ejemplifican rutinas con temáticas y hábitos propios de la cultura costarricense. 
Por tanto, el abordaje de criterios pedagógicos para la atención de la diversidad, al igual que se ha reiterado en otros párrafos de este documento, permiten sugerir que "el trato igualitario" fue considerado como la estrategia y el criterio prevaleciente en la docente, para trabajar y evaluar a los infantes nicaragüienses. Este concepto subyacente de trato igualitario obligó a la investigadora a consultar directamente a la docente sobre su concepto de educación para la diversidad, especialmente, porque este concepto y a la vez principio, se relacionaron con las implicaciones de una ideología asimilacionista.

Frente a estas consultas realizadas a la docente N:

¿Cuál es su concepto de educar en la diversidad cultural y para esta?

¿Cómo debe abordarse la diversidad cultural en el salón de clases?

Ella respondió:

Docente N: "Bueno yo salí hace muchos años de la universidad y ahora no tengo mucha información de lo nuevo, pero si es de los niños nicaragüenses yo creo que ellos tienen derecho a la educación y que se les debe ayudar para que conozcan y se adapten a nuestro país, porque como ellos viven aquí deben adaptarse".

"Yo creo que todos los niños son iguales, para mí los nicaragüenses y los costarricenses no tienen diferencias, todos son buenos, amables y cariñosos, no veo ¿porqué uno deba establecer diferencias en el trato y trabajo con ellos?

(Extracto de observación 14).

Estas respuestas de la docente $\mathrm{N}$ y sus acciones en la práctica, evidenciadas en los protocolos de observación, sugieren que la ilusión del trato igualitario como estrategia para el abordaje de la diversidad cultural es la principal acción que utilizó la docente participante en el estudio, para el abordaje de la diversidad cultural. Esta ilusión del trato igualitario es considerada como otra de las creencias implícitas de la maestra en relación con el desarrollo de la niña y los niños nicaragüenses.

En el concepto que subyace de la práctica docente, relacionado con el trato igualitario, se evidencia que la docente contrapone su ilusión profesional, entendida como lo que ella espera que sea el trato igualitario, con lo establecido en la teoría de los pilares de la educación intercultural que se refieren a "(...) no jerarquizar las culturas, hablar de diferencias y no de desigualdades, y valorizar las diferentes culturas como fuente de enriquecimiento y de desarrollo" (Devalle y Vega, 1999, p. 32).

Después de analizar la evaluación en el contexto de la práctica docente, es importante retomar evidencias de un patrón establecido con la disciplina. Esto con el fin de establecer las estrategias más utilizadas por la maestra y su relación con el concepto subyacente de trato igualitario.

\subsection{El grito: una estrategia para disciplinar a la niña y los dos niños nicaragüenses}

Según Sabariego (citada por Essomba et al., 1999), el tratamiento de la diversidad cultural desde una perspectiva intercultural sugiere la necesidad de considerar aspectos tales como el clima de aprendizaje, el cual debe caracterizarse por ser respetuoso y donde se reconocen y aceptan las diferentes culturas en el aula. Además, indica la autora que los procesos de enseñanza y aprendizaje deben realizarse en un marco dialógico y con más refuerzos positivos que negativos. 
En términos de la práctica docente, las observaciones permiten confirmar que las normas de comportamiento en el aula fueron impuestas por la docente $\mathrm{N}$, quien en todo momento utilizó un tono de voz fuerte y en ocasiones el vocabulario fue agresivo (necios, yegüitas, malcriados, insoportables) para llamar la atención de los niños y niñas, tanto de los nicaragüenses como de los costarricenses.

Por otra parte y siempre relacionado con el concepto de disciplina, las niñas y los niños nicaragüenses y costarricenses fueron regañados por la docente, debido a múltiples razones: porque no recogen como ella quiere, porque no están en el área que deben, porque no le hacen caso, porque los carros no se pueden utilizar para ir al recreo, porque tocaron los bolsos, porque hablan y no lo tenían que hacer, porque a una de las niñas se le cayeron las cosas, porque pelean con el compañero y lo maltratan; en síntesis, los regaños y el tono de voz fuerte acompañó la rutina de trabajo de la docente. Situación que es confirmada y, en cierto modo rechazada, por los niños y las niñas, quienes en el siguiente cuadro expresaron, en su mayoría, que quisieran que su maestra no les grite, no los regañe, ni los maltrate.

Cuadro 2

Acciones que los niños y las niñas nicaragüenses y costarricenses sugieren que su maestra no realice en el salón de clases

\begin{tabular}{lcc}
\hline Categorías & Frecuencia & Porcentaje \\
\hline Regañe & 12 & $46 \%$ \\
Grite & 10 & $38 \%$ \\
Maltrate & 3 & $12 \%$ \\
Fuera mi maestra & 1 & $4 \%$ \\
Total & 26 & $100 \%$ \\
\hline
\end{tabular}

Nota. Entrevista aplicada a niños y niñas nicaragüenses y costarricenses participantes en la investigación.

Esta estrategia de regaños y gritos como una alternativa para mantener al grupo disciplinado fue, según la docente $\mathrm{N}$, una forma de responder al ambiente de trabajo cuando tuvo la responsabilidad de atender a muchos niños y niñas, simultáneamente.

Docente N: "Yo creo que no es bueno que uno grite, pero es que, a veces, los chiquillos lo sacan a uno de sus casillas, especialmente, cuando los días no son tan buenos, pero creo que se puede mejorar, especialmente, si a ellos no les gusta, pero que difícil, el próximo año voy a trabajar con modificadores de conducta"

Conversación informal con la docente No. 4

La frase que expresa la docente, si bien es cierto indica una concientización por su parte hacia un trato menos agresivo con los niños, la realidad mostró que los cambios no fueron evidentes y que la práctica del grito y el regaño se mantuvo hasta finalizar el curso lectivo. Esta forma de trabajar con la niña y los niños nicaragüenses demostró ser, también, igual para los niños y las niñas costarricense; es decir, los extranjeros no fueron segregados o bien maltratados como población única; sin embargo, la docente $\mathrm{N}$ en varias ocasiones comentó a la investigadora acerca 
de su preocupación por el nivel de agresividad de uno de los infantes nicaragüenses; pero en las observaciones realizadas se logró determinar que $\mathrm{S}$ era un niño activo, efusivo, que en ocasiones mantuvo peleas y discusiones con sus compañeros, pero sus niveles de agresividad no fueron superiores a los de R, el otro niño nicaragüense.

En términos generales, es impreciso hablar de un concepto de procesos de enseñanza y aprendizaje dialógicos, cargados de refuerzos positivos, cuando en realidad la práctica docente sugiere constantes regaños y gritos como la alternativa para tratar de mantener el control del salón de clases y el dominio sobre la niña y los dos niños nicaragüenses.

Por otra parte, en este contexto no se puede hablar tampoco de un respeto por los aprendizajes de las culturas diversas, especialmente, porque la relación docente-alumno se basó en el poder de dominación, como concepto subyacente de una práctica que nuevamente demuestra cualidades o asociaciones interesantes que no se relacionan con una propuesta de carácter intercultural, por medio de la cual, según Sabariego (citada por Essomba et al. 1999), se deben favorecer un proceso de enseñanza-aprendizaje dialógico, interactivo y con más refuerzos positivos que negativos y en la resolución de conflictos se debe utilizar el diálogo crítico y el debate como medidas preferentes.

El tema a analizar en los siguientes párrafos será la metodología, debido no solo a que forma parte de la práctica docente, sino a que, además, evidencia el cómo se concretan en la realidad los procesos de enseñanza y aprendizaje.

\subsection{La interculturalidad, una propuesta alejada de la realidad metodológica del aula}

Desde la perspectiva de la autora Sabariego (citada por Essomba et al. 1999), un proyecto de educación intercultural exige, necesariamente, la existencia de varios aspectos que se relacionan con la metodología docente y que se refieren a:

a. ¿Se concede atención a la expresión de la multiculturalidad en el ambiente material del aula?

b. ¿El clima de aprendizaje es respetuoso, reconoce y acepta las diversas culturas presentes en el aula (fomentando la valoración positiva de la diversidad, así como las actitudes de respeto, tolerancia y solidaridad entre el alumnado)?

c. ¿Es posible la expresión espontánea de la propia identidad cultural? ¿Se permite que esta expresión se legitime, se valore y se analice críticamente? ¿Se permite construir la propia identidad cultural de un modo enriquecedor adquiriendo, al mismo tiempo, competencia para tener conciencia de los esquemas culturales que hay alrededor?

d. ¿Las programaciones y la selección de contenidos y materiales se efectúan con criterios de diversidad cultural?

e. ¿Se facilita la expresión oral de las vivencias culturales y de los saberes prácticos del alumnado?

f. ¿El modelo educativo que existe en el aula estimula aquellas metodologías que posibilitan el marco de interrelaciones (es decir, propuestas que favorezcan la interacción entre iguales, el trabajo cooperativo, las dinámicas comunicativas, el intercambio de roles o las tareas colectivas, de cara a facilitar el descubrimiento de la dimensión positiva del otro y fomentar relaciones interculturales constructivas)?

g. ¿Los recursos metodológicos que se utilizan permiten el tratamiento adecuado de la diversidad (la adaptación al ritmo de aprendizaje y el seguimiento personalizado del 
alumnado mediante una organización flexible del tiempo y la diversificación de técnicas, modalidades de trabajo, materiales, así como de actividades de aprendizaje para la adquisición de un mismo contenido y de contenidos para un mismo objetivo)?

h. ¿Se favorece un proceso de enseñanza-aprendizaje dialógico, interactivo y con más refuerzos positivos que negativos?

i. ¿En la resolución de conflictos se utilizan el diálogo crítico y el debate como medidas preferentes? (p. 30-34)

Durante el proceso de observación en el aula, la investigadora identificó algunos de los aspectos propuestos por Sabariego (citada por Essomba et al. 1999) y que se refieren a la metodología de aula; sin embargo, en la mayoría de los casos los puntos expuestos por este autor se distancian de lo que podría considerarse como un ambiente de aprendizaje coherente con los principios y objetivos de una propuesta de educación intercultural.

La metodología empleada por la docente en el salón de clases estuvo determinada por relaciones interpersonales niño-docente, bajo criterios de dominio de la maestra hacia los infantes, además, los discentes nicaragüenses en todo momento demostraron relaciones cooperativas de juego con sus iguales. Si bien es cierto, el juego estuvo presente en el aula como una actividad de gran predilección para la niña y los dos niños nicaragüenses, la docente $\mathrm{N}$ se ausentó de dichos juegos, tanto los realizados dentro, como fuera del salón de clases, debido a la atención excesiva que ella le brindó a cuestiones de índole administrativa.

Si bien es cierto, la docente estuvo muy ausente en los momentos de juego, entre los niños, esta actividad cobró gran sentido en la mayoría de las actividades y provocó contacto físico permanente entre los niños y las niñas nicaragüenses y costarricenses, situación que se consideró como positiva de cara a la incorporación de una propuesta intercultural, la cual, según Besalú (2002), uno de los principios básicos constituye el contacto físico entre los grupos, es decir, no comparte la visión de separar a las personas e integrarlas en algunos momentos del día.

Por otra parte, la estrategia que siguió la docente $\mathrm{N}$ para la organización y desarrollo de las actividades consistió en lo que la investigadora denominó "uno por uno", debido a que, para la mayoría de las actividades, tales como distribución de los niños y las niñas en las áreas de trabajo, así como también, para la merienda y el trabajo en apresto, o en arte, la docente $\mathrm{N}$ recurrió al envío de niño por niño y niña por niña como una estrategia de orden, que de alguna manera causa que los infantes deban esperar periodos amplios para ser llamados e iniciar la actividad.

Docente N: "A ver es hora de ir a las áreas".

Niños y Niñas: Gritan y levantan la mano, abalanzándose sobre la docente para solicitar el área a la cual desean tener acceso para jugar.

Docente N: "Que necios no se pueden esperar, parecen animalitos".

La docente busca una hoja para anotar el área a que irán los niños y las niñas.

Docente N: "Luis, usted vaya a casit;, Pablo, usted a construcción" (Así continúa mencionando cada uno de los nombres de los niños y niñas, hasta que concluye, la actividad de envío a las áreas, tarda aproximadamente 15 minutos de los 45 que se supone durará el periodo).

Extracto de observación 8 
En lo relativo a la estructura del trabajo diario, la rutina se desarrolló en varios periodos que se refirieron a: actividades iniciales, conversación, juego trabajo, merienda, recreo; si bien es cierto, la música y la literatura estuvieron incluidas en el documento escrito del horario, en la práctica su ejecución fue muy poco frecuente. Sin embargo, estos cambios en la rutina de horario, en todo momento, fueron competencia de la docente $\mathrm{N}$ y en la mayoría de los casos se recargó tiempo extra al periodo de juego trabajo en detrimento de los otros.

Relacionado con la información anterior, los contenidos y tipos de actividades empleadas en cada periodo se caracterizaron por una ausencia importante de los intereses y necesidades de los infantes nicaragüenses; esto es, la presencia de la cultura extranjera no se evidencia en propuestas de juegos o temáticas analizadas, por tanto, el espacio para la expresión de la propia cultura en realidad fue muy reducido.

El último elemento a analizar en relación con la metodología se refiere a la atención individualizada que recibe la niña y los niños nicaragüenses, por parte de su docente. Dicha atención fue realmente escasa, ya que se redujo al horario establecido para dicho periodo, por ejemplo, dentro de la rutina de trabajo diario, cuando los infantes requirieron ayudas individuales para realizar sus juegos, ellos acudieron a los compañeros o bien a la docente, quien se ubicó con frecuencia en su escritorio.

La metodología que utilizó la docente $\mathrm{N}$ en su práctica de trabajo diario implicó una serie de conceptos subyacentes y creencias tales como la carencia de estrategias metodológicas tendientes al abordaje de la cultura nicaragüense. Esto debido a que no se seleccionan materiales, recursos, actividades y contenidos que permitan a la niña y los dos niños expresar sus formas de vida y cultura en general.

Por el contrario, el aula estuvo cargada de espejismos costarricenses reflejados en los juegos, tareas, apresto, actividades y formas de relacionarse, que en todo momento se convirtieron en verdugos del otro, apoderándose de la cultura extranjera, reduciéndola hasta convertirla en un elemento prácticamente nulo, alejado del discurso y de las buenas intenciones de la docente $\mathrm{N}$, quien en todo momento mostró gran sensibilidad hacia los problemas económicos que presentaron esta niña y los dos niños, pero no demostró, en su práctica educativa, los principios de una propuesta intercultural o multicultural; más bien, la existencia del principio de dominación y relaciones de poder sugieren el paso de esta práctica por la ideología asimilacionista, cuyo objetivo primordial radica en absorber a las minorías.

En este caso particular, el manejo de la minoría (niña y niños nicaragüenses) se ejerció de una forma inclusive inconsciente por parte de la docente N. Sus actitudes de trato igualitario como estrategia para integrar a esta niña y estos dos niños evidencian una relación de afecto e inclusive "respeto" que, desde la perspectiva de la maestra, se mantiene cuando no se establecen diferencias entre los niños porque todos "son iguales".

\section{Conclusiones}

El trabajo de campo, que exigió un largo proceso de observación, entrevistas, devoluciones de información, entre otros, permite a la investigadora plantear las siguientes conclusiones

a. En la práctica de aula la maestra no demuestra un trato diferenciado entre niños nicaragüenses y costarricenses, lo que hace pensar que el trato igualitario es la estrategia "per se" que utiliza la docente para resolver el tema de la diversidad cultural en el aula. 
b. En términos de la planificación, los datos permiten concluir que los intereses y necesidades de niñas y niños nicaragüenses y costarricenses no se introducen dentro de la organización del trabajo diario, asimismo, la introducción de temáticas relativas a la cultura extranjera fueron inexistentes.

Por otra parte, el planeamiento de trabajo diario se ejecuta con criterios pedagógicos de una atención a la diversidad sumida en el principio o ilusión del "trato igualitario", como la alternativa más viable que utiliza la docente para abordar el tema de la diversidad cultural en el aula.

c. Los datos son claros en demostrar que los materiales y rutinas de trabajo diario no facilitan el intercambio cultural entre niños nicaragüenses y costarricenses, asimismo, estas rutinas, en términos de horarios, evidencian poca flexibilidad para la participación activa de los niños y de las niñas de las diferentes nacionalidades.

d. La disciplina es asumida por la docente, en su práctica de trabajo diario, como un asunto que se aborda con criterios de regaño, utilizando tonos de voz fuerte y vocabulario agresivo (como ejemplo: necios, cochinas, etc.).

e. Los datos son concluyentes en demostrar que la metodología utilizada por la docente se caracteriza por la verticalidad en las relaciones, es decir, la maestra es quien propone y ejecuta, mientras que los niños y niñas nicaragüienses y costarricenses responden a los estímulos del trabajo diario. Asimismo, la estrategia "uno por uno", puesta en práctica por la docente para organizar y desarrollar las diferentes actividades, hace suponer que, definitivamente, existe un concepto de aprendizaje basado no en el dinamismo y la autonomía del infante, sino, más bien, en la espera y en la asimilación de instrucciones.

f. En términos generales, la práctica docente de la maestra participante en el estudio muestra características que evidencian una relación con la perspectiva teórico-práctica asimilacionista, esto es, se establecen relaciones de poder maestro-alumno que generan una dominación del primero hacia el segundo. Asimismo, el trato igualitario que reciben los infantes nicaragüienses y costarricenses evidencia criterios pedagógicos asociados que no exploran en la diversidad cultural la riqueza de un aprendizaje basado en el respeto por el otro.

\section{Referencias bibliográficas}

Aguado, T., Gil, I. y Mata, P. (2008). El enfoque intercultural en la formación del profesorado. Dilemas y propuestas. Revista Complutense de Educación, 19(2), 275-292. Reuperado de http://revistas.ucm.es/edu/11302496/articulos/RCED0808220275A.PDF

Ander-Egg, E. (2001). Los desafios de la educación en el siglo XXI. Algunas reflexiones sobre los retos del futuro inmediato. San Fe, Argentina: Homo Sapiens Ediciones.

Besalú, X. (2002). Diversidad Cultural y Educación. Madrid: Editorial Síntesis, S.A.

Bueno, J. (1997). Controversias en torno a la educación multicultural. Recuperado de www.lie.upn. mx/docs/Diplomados/LineaInter/Bloque2/.../Lec2.pdf

Devalle, A., y Vega, V. (1999). Una escuela en y para la diversidad. El entramado de la diversidad. Sao Pablo, Brasil: Editorial AIQUE. 
Essomba, M. [Coord.], Barandica, E., Sabariego, M., Sandin, M. P., del Campo, J., Viñas, J. et al. (1999). Construir la escuela intercultural. Madrid: Editorial Graó.

Jordán, J. A. (1994). La escuela multicultural. Un reto para el profesorado. Barcelona: Paidós.

Luft, J. (1999, Febrero). Teachers' salient beliefs about a problem-solving demonstration classroom in-service program [Creencias que sobresalen en los docentes con respecto a la demostración en el aula de resolución de problemas en programas de servicio]: Journal of Research in Science Teaching, 36(2), 141-158. doi: 10.1002/(SICI)1098-2736(199902)36:2<141::AIDTEA3>3.0.CO;2-P

Lumpe, A., Haney, J., y Czerniak, C. (2000, Marzo). Assesing Teachers' Beliefs about Their Science Teaching Context [Evaluación de las creencias de los docentes en relación con su contexto de enseñanza de las ciencias]. Journal of Research in Science Teaching, 37(3), 275-292. doi: 10.1002/(SICI)1098-2736(200003)37:3<275::AID-TEA4>3.0.CO;2-2

Maxwell, J. (1996). Qualitative Research Desing an Interactive Approach. United Estates of America: SAGE Publications.

Pajares, F. (1992, Fall). Teachers' Beliefs and Educational Research: Cleaning Up a Messy Construct [Creencias del docente e investigación Educativa: corrigiendo un concepto mal construido]. Review of Educational Research, 62(3), 307-332. doi: 10.3102/00346543062003307

Spanneberg, R. (2001). Teachers' Beliefs and Practices related to a Professional Development Programme in South African Rural Schools. En línea: http://www.aare.edu.au/01pap/ spa01178.htm

Schuck, S. (1997, Julio). Using a research simulation to challege prospective teachers' beliefs about mathematics [Utilización de una simulación de investigación para retar las creencias propuestas de maestros acerca de las matemáticas]. Teaching and Teacher Education, 13(5), 529-539. doi:10.1016/S0742-051X(97)85541-2 